\title{
Physical Restrictions to Cosmological Gamma-Ray Burst Models ${ }^{\star}$
}

\author{
G.S. Bisnovatyi-Kogan
}

Space Research Institute, Russian Academy of Science, Profsoyuznaya 84/32, Moscow 117997, Russia

gkogan@mx.iki.rssi.ru

Summary. The present common view about GRB origin is related to cosmology, and is based on statistical analysis, and on measurements of the redshifts in the GRB optical afterglows of long GRB. No correlation is found between redshifts, GRB spectrum, and total GRB fluence. Comparison of KONUS and BATSE data about statistics and hard X-ray lines is done, and some differences are noted. Hard gamma-ray afterglows, prompt optical spectra, hard X-ray lines, polarization measurements could be very important for farther insight into GRB origin. Possible connection of short GRB with soft gamma repeaters is discussed.

\section{GRB Models}

It is generally accepted now that cosmic gamma-ray bursts (GRB) have a cosmological origin. The first cosmological model, based on explosions in active galactic nuclei (AGN) was suggested in [25]. A mechanism of the GRB origin in the vicinity of a collapsing object based on neutrino-antineutrino annihilation was analyzed in [5]. GRB production in supernova explosion was discussed in [7]. The GRB models may be classified by two levels. The upper one is related directly to the observational appearance, and include 3 main models" 1) Fireball, 2) Cannon Ball, and 3) Precessing Jets.

The main restrictions are connected with the next (basic) level of GRB model, which is related to energy source, producing a huge energy output necessary for a cosmological GRB model. This class contains 5 main models:

1. $(\mathrm{NS}+\mathrm{NS}),(\mathrm{NS}+\mathrm{BH})$ mergers. This mechanism was investigated numerically in $[27,28]$. Gamma radiation is produced here by $(\nu, \tilde{\nu})$ annihilation, and the energy output is not enough to explain most powerful GRB even with account of strong beaming. The energy emitted in the isotropic optical afterglow of GRB $990123[1,18]$ is about an order of magnitude larger than the total radiation energy output in this model.

2. Magnetorotational explosion. Magnetorotational explosion, proposed in [23] for a cosmological GRB, had been suggested earlier for the supernova

* This work is partly supported by RFFI grant 02-02-16900, INTAS/ESA grant 99-120, INTAS grant 00-491 
explosion in [6]. Numerical calculations gave the efficiency of a transformation of the rotational energy into the kinetic one at the level of few percent $[3,4]$. This is enough for the supernovae energy output, but is too low for cosmological GRB.

3. Hypernova. This model, suggested in [23] is popular now, because traces of the supernova explosions are believed to be found in the optical afterglows of several GRB $[13,35]$. The hypernova model is based on a collapse of a massive core, formation of a black hole $M_{b h} \sim 20 M_{\odot}$, surrounded by a massive disk with a rapid accretion and appearance of GRB [19]. This model seems to be most promising now.

4. Magnetized disks around rotating (Kerr) black holes ( $\mathrm{RBH}$ ). This model is based on extraction of rotating energy of $\mathrm{RBH}$ when magnetic field is connecting the $\mathrm{RBH}$ with the surrounding accretion disk or accretion torus [37].

5. A model proposed in [29] creates the GRB by the pair-electromagnetic pulse from an electrically charged black hole surrounded by a baryonic remnant. The main problem here is how to form such a strongly charged BH.

\section{Basic Data}

\subsection{Statistics}

Statistical arguments in favor of the cosmological origin of GRB are based on a visual isotropy of GRB distribution on the sky in combination with a strong deviation of $\log N-\log S$ distribution obtained in BATSE observations [22] from the euclidian uniform distribution with the slope $3 / 2$. Similar properties have been obtained in KONUS experiment [20] where the authors explained deviations from $3 / 2$ slope by selection effects. The analysis of KONUS data with account of selection effects made in [17] gave the average value $\left\langle V / V_{\max }\right\rangle=0.45 \pm 0.03$; the value 0.5 corresponds to pure uniform distribution. KONUS data had been obtained in conditions of constant background. Similar analysis [31] of BATSE data, obtained in conditions of substantially variable background, gave resulting $\left\langle V / V_{\max }\right\rangle=0.334 \pm 0.008$. These two results seems to be in contradiction, because KONUS sensitivity was only 3 times less than that of BATSE, where deviations from the uniform distribution in BATSE data are sill large [15].

Statistical analysis and of BATSE data, divided in 4 classes according to their hardness and calculation of $\left\langle V / V_{\max }\right\rangle$ for different classes have been done in [32]. In the cosmological model we may expect smaller value of $<V / V_{\max }>$ for softer GRB in the case of a uniform sample, because larger red shifts would correspond to softer spectra. The result is quite opposite, and soft GRB have larger $\left\langle V / V_{\max }>\right.$ than the hard ones, 0.47 and 0.27 
respectively. It is supposed in [32] such a strong excess of luminosity in hard GRB, which overcomes the tendency of the uniform sample.

The influence of statistical errors in presence of the threshold was analyzed in [8]. The $\log N-\log S$ curve in presence of statistical errors on the level of average 10 thresholds has a similarity with the BATSE distribution.

\subsection{Optical Afterglows and Red Shifted Lines}

The spectra of optical afterglows have shown large red shifts $z$, up to 4.5 , indicating to the cosmological origin of GRB and their enormous energy outputs. In most cases the red shifts have been measured in the faint host galaxies. The list of red shift measurements is given in $[10,14]$. The redshift data from $[14]$ are listed in [10] by total GRB fluences. Huge energy output during a short time $(0.1$ - few 100 seconds) create problems for the cosmological interpretation.

\subsection{Collimation}

To avoid a huge energy production, strong collimation is suggested in the radiation of GRBs. The restriction to the collimation angle follows from the analysis of the probability of appearance of the orphan optical afterglow [26], which should have lower or no collimation. The absence of any variable orphan afterglow in a search poses restriction $\Omega_{\text {opt }} / \Omega_{\gamma}<<1 / 2100$. At radio wavelengths published source counts and variability studies have been used in [24] to place a limit on the collimation angle, $\theta_{\gamma} \geq 5^{\circ}$.

Comparison of the red shifts and fluences presented in [10] shows no correlation between distance and observed flux (see Fig. 1). It is explained by strong collimation, and strong scattering is connected with different sight angles in the beam. If the collimation is connected with the relativistic bulk motion [13], then strong correlation between GRB duration and their power should exist: stronger GRB should be shorter. Absence of such correlation excludes models based on the relativistic bulk motion collimation.

\subsection{Prompt Optical Afterglows}

The prompt optical afterglow of GRB 990123 was caught by optical observations 22 seconds after the onset of the burst [1,2]. GRB 990123 was detected by BATSE on 1999 January 23.407594. The event was strong and lasted $\geq 100 \mathrm{~s}$. The T50 and T90 durations are $29.82( \pm 0.10) \mathrm{s}$ and $63.30( \pm 0.26)$ $\mathrm{s}$, respectively. The maximum optical brightness $8.95^{\mathrm{m}}$ was reached $30 \mathrm{~s}$ after the GRB beginning, and after $95 \mathrm{~s}$ it was already at $14.5^{\mathrm{m}}$. So the gamma ray maximum almost coincides with the optical one. The observed optical luminosity, related to the redshift $z=1.61$ reaches $L_{\text {opt }} \approx 4 \cdot 10^{49} \mathrm{ergs} / \mathrm{s}$, what is about 5 orders of magnitude brighter than optical luminosity of any 


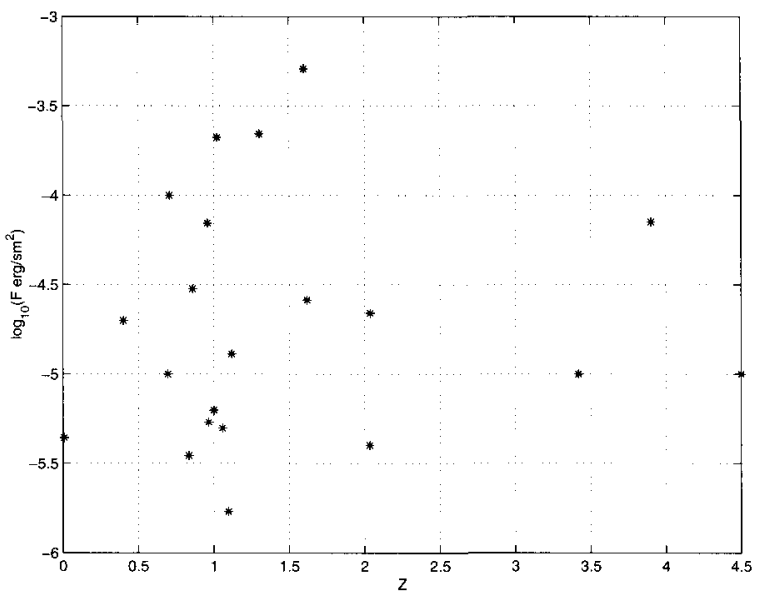

Fig. 1. Fluence $F$ versus redshift $z$ for GRB data from [10].

observed supernova. The energy of the prompt optical emission reaches $10^{51}$ ergs, and the isotropic gamma-ray flux is about $2.3 \cdot 10^{54}$ ergs, what exceeds the rest energy of the Sun $[1,18]$. Another bright afterglows have been observed in GRB $021004\left(15^{m}, \mathrm{z}=2.3\right)$, GRB $030329\left(12.4^{m}, \mathrm{z}=0.168\right)$ and GRB $030418\left(16.9^{m}\right)$. Brightest visual magnitude and redshift are given in brackets. Among those, the most remarkable afterglow observed by many observatories was in GRB 030329 (see e.g. [12, 30]), where the supernova was probably detected by the features of the optical spectra [36].

\subsection{High-energy Afterglow}

EGRET observations on CGRO have shown that GRB emit also very hard gamma photons up to $20 \mathrm{GeV}[15]$. The number of GRB with detected hard gamma radiation is about 10 , from them 5 bursts had registered photon energies over $100 \mathrm{MeV}$ [33]. Hard gamma emission, as a rule, continues longer than the main (soft) gamma ray burst, up to 1.5 hours in the GRB940217. Comparison of the angular aperture of EGRET and BATSE leads to conclusion that hard gamma radiation could be observed in large fraction (about one half) of all GRB. Spectral slope in hard gamma region lays between (2 ) and (-3.7), and varies rapidly, becoming softer with time (GRB920622 in $[34])$.

\subsection{Hard X-ray Lines}

Hard gamma-ray lines in GRB spectra had been discovered by KONUS [21]. They had been interpreted there as cyclotron lines, and have been seen in 20$30 \%$ of the GRB. These spectra had shown a distinct variability: the visible 
absorption decreases with time. In BATSE data [11] 13 statistically significant line candidates have been found from 117 GRBs. The only interpretation of hard spectral feature in the cosmological model [16] is based on the blueshifted $(\Gamma=25-100)$ spectrum of the gas cloud illuminated by the gamma radiation of the fireball, and seems rather artificial.

\section{Discussion}

The investigation of orphan optical bursts by all-sky optical monitoring could be useful for putting better limits for the collimation. It is important to obtain prompt optical spectra of the GRB afterglows when the optical counterpart is still luminous, to investigate the polarization of the optical and X-ray afterglow for clarification of the radiation mechanism, and get more data on the hard gamma-ray afterglows.

The statistical analysis reveals at least two separate samples consisting of long ( $>\sim 2 \mathrm{~s}$ ) and short bursts. Optical afterglows and redshift measurements have been done only for long bursts. Therefore, it is not excluded that short bursts have different (may be galactic) origin. It is interesting to compare the properties of short GRB with giant bursts from soft gamma-repeaters (SGR) inside the Galaxy. From the larger distance only giant bursts would be registered, which could be attributed to the short GRB. The existence of the giant bursts in the SGR (3 in 4 firmly known SGR in the Galaxy and LMC) implies a possibility for observation of giant bursts, which appear as short GRB, in other neighboring galaxies. The estimation gives more than 10 expected "short GRB" of this type from M 31 and other close neighbors [9]. The absence of any GRB projecting on the local group galaxies may indicate that SGR are more close and less luminous objects, than it is now accepted $[9]$.

\section{References}

1. C.W. Akerlof et al. : Nature 398, 400 (1999)

2. C.W. Akerlof, T.A. McKay: GCN 205 (1999)

3. N.V.Ardeljan, G.S. Bisnovatyi-Kogan, S.G. Moiseenko: Physics-Uspekhi 40, $1076(1997)$

4. N.V. Ardeljan, G.S. Bisnovatyi-Kogan, S.G. Moiseenko: Astron. Astrophys. 355, $1181(2000)$

5. V.S. Berezinsky, O.F. Prilutsky,: Astron. Astrophys. 175, 309 (1987)

6. G.S. Bisnovatyi-Kogan: Sov. Astron. 14, 652 (1971)

7. G.S. Bisnovatyi-Kogan, V.S. Imshennik, D.K. Nadyozhin, V.M. Chechetkin: Astrophys. Space Sci. 35, 23 (1975)

8. G.S. Bisnovatyi-Kogan: Astron. Astrophys. 324, 573 (1997)

9. G.S. Bisnovatyi-Kogan: Mem. Soc. Astron. It. 73, 318 (2002)

10. G.S. Bisnovatyi-Kogan: astro-ph 0310361 (2003) 
11. M.S. Briggs et al. : astro-ph 9901224 (1999)

12. R. Burenin et al. : GCN 2001 (2003)

13. S. Dado, A. Dar, A. De Rújula: Astron. Astrophys. 388, 1079 (2002)

14. S.G. Djorgovski et al. : astro-ph 0107535 (2001)

15. G.J. Fishman, C.A. Meegan: Ann. Rev. Astron. Astrophys. 33, 415 (1995)

16. C.J. Hailey, F.A. Harrison, K. Mori: Astrophys. J. Lett. 520, L25 (1999)

17. J.C. Higdon, M. Schmidt: Astrophys. J. 355, 13 (1990)

18. S. Kulkarni et al. : Nature 398, 389 (1999)

19. A.I. MacFadyen, S.E. Woosley: Astrophys. J. 524, 262 (1999)

20. E.P. Mazets et al. : Sov. Astron. Lett. 6, 318 (1980)

21. E.P. Mazets et al. : Astrophys. Space Sci. 82, 261 (1982)

22. C. Meegan et al. : Nature 355, 143 (1992)

23. B. Paczynski: Astrophys. J. Lett. 494, L45 (1998)

24. R. Perna, A. Loeb: Astrophys. J. Lett. 509, L85 (1998)

25. O.F. Prilutsky, V.V. Usov: Astrophys. Space Sci. 34, 387 (1975)

26. J.E. Rhoads: astro-ph 0103028 (2001)

27. M. Ruffert, H.-Th. Janka: Astron. Astrophys. 338, 535 (1998)

28. M. Ruffert, H.-Th. Janka: Astron. Astrophys. 344, 573 (1999)

29. R. Ruffini et al: Astron. Astrophys, 359, 855 (2000)

30. V. Rumjantsev et al. : GCN 2005 (2003)

31. M. Schmidt: Astrophys. J. Lett. 523, L117 (1999)

32. M. Schmidt: astro-ph 0101163 (2001)

33. E.J. Schneid et al. : NY Acad. Sci. Ann. 759, 421 (1995)

34. E.J. Schneid et al. : Astrophys. J. 453, 95 (1995)

35. V.V. Sokolov: astro-ph 0102492 (2001)

36. K.Z. Stanek et al. : astro-ph 0304173 (2003)

37. M.H.P.M. van Putten: Phys. Rep. 345, 1 (2001) 